Manuelle Medizin 2017 · 55:331-332

https://doi.org/10.1007/s00337-017-0341-x

(c) Springer Medizin Verlag $\mathrm{GmbH}$, ein Teil von Springer Nature 2017

CrossMark

\section{W. von Heymann}

Bremen, Deutschland

\title{
Erziehung zur Selbstreflexion
}

\section{Ein Meilenstein zur Entwicklung eines kompetenten, professionellen Manualmediziners}

"Continuing medical education“ (CME), lebenslanges Lernen für Ärzte - wer kennt das nicht, wer hat sich noch nicht über die oft unsinnigen und meist bürokratischen Regularien dieses Systems geärgert? Ursprünglich ist die kontinuierliche ärztliche Fortbildung eine ethische Verpflichtung für jeden Arzt, die jedoch zunehmend in bürokratisch kontrollierte Fortbildungszwänge mündet, weil ohne die entsprechenden „Punkte" plötzlich Abrechnungsmöglichkeiten gesperrt werden können. Warum mussten diese Zwänge eingeführt werden? Wahrscheinlich, weil die Angebote zum Erwerb der „Punkte“ langweilig und schlecht waren, weil es keinen inhaltlichen Anreiz dafür gibt - weil an diesen Angeboten eben nichts Verlockendes war.

Wie kann denn nun eine interessante und effektive Weiter- und Fortbildung in der manuellen Medizin aussehen? Über diese didaktische Herangehensweise wollen wir uns in diesem Heft Gedanken machen. Sollte wirklich Edukation, also das Lernen von Fakten, unser Ziel sein? Dieser Begriff ist wohl etwas unglücklich, denn Edukation findet im Studium statt. Daher kennt der frisch approbierte Arzt unter allen Ärzten die meisten Fakten. Ist er daher auch der beste? Sicher nicht - die wirkliche Ausbildung einer professionellen ärztlichen Persönlichkeit beginnt trotz technischer Dummys, sog. Bedside-Teaching und praktischem Jahr erst nach der Approbation. Diese Fort- und Weiterbildungen sollten daher eher unter dem Begriff der kontinuierlichen professionellen Entwicklung („continuing professional development“,
CPD) durchgeführt werden. Es gilt also, etwas anderes anzubieten als das Lernen weiterer Fakten.

Im Prinzip kennen die Kollegen, die sich zu den Weiterbildungskursen anmelden, die Anatomie und Neurophysiologie schon aus der Vorklinik, sie kennen die anatomischen Pathologien und die Pathophysiologie aus den klinischen Semestern und dem praktischen Jahr und sie kennen viele Fußnoten über seltene Pathologien. Aber sie haben ein funktionelles Verknüpfen dieser Fakten für den praktischen Alltag nicht gelernt, sie wissen noch nicht, wie der konkrete individuelle Patient in seiner persönlichen Situation abzuholen und zu führen ist. Sie können eine Bildgebung beschreiben, kennen aber die Bandbreite der klinischen Relevanz noch nicht und vertrauen der Technik mehr als ihren eigenen Händen. Hier gilt es, in den Weiterbildungskursen die funktionellen Verknüpfungen aufzuzeigen und die Hände in ihrer Palpationsfähigkeit so zu trainieren, dass sie am Ende der Weiterbildung zur gleichen „Reliabilität“ der Palpationsbefundung befähigt sind wie die erfahrenen Manualmediziner. Auch sollten sie durch das Denken in den Aspekten von funktioneller Störung und strukturellem Schaden, unterstützt durch gezielte Befragung, in der Lage sein, den jeweiligen Kern der Beschwerden zu verstehen und damit eine Diagnose rational zu finden sowie dem Patienten verständlich und überzeugend eine optimale Therapie vorzuschlagen.

In der ärztlichen Fort- und Weiterbildung hat sich jedoch auch gezeigt, dass Wissen und praktische Kenntnisse noch nicht das Ende der professionellen Entwicklung darstellen. Natürlich bedarf es immer wieder des Angebots praktischer („hands-on“) Refreshing-Kurse gegen die Angst vor einer falschen Manipulationstechnik. Diese Angebote sollten ausgebaut werden. In einer von der Schweizerischen Ärztegesellschaft für Manuelle Medizin 2009 durchgeführten internen Befragung zugelassener und aktiver Manualmediziner, die täglich manuell diagnostizieren und behandeln, gaben nur $16 \%$ an, dass sie sich auch täglich therapeutisch-manipulativ an die Halswirbelsäule herantrauen. Dies ist ein beschämendes Zeugnis für die didaktische Qualität unserer praktisch ausgelegten Kurse. Hier brauchen wir Konzepte, das praktische Training der Kollegen zu verbessern. Allerdings kann auch professionelles ärztliches Verhalten zur Identifizierung und ggf. auch die angemessene Therapie psychosomatischer Störungen nicht in Manipulationskursen („Knack-Kurse“) vermittelt werden. Eine solche Formung der ärztlichen Persönlichkeit ist immer das Ergebnis einer reflektierten Selbsterfahrung unter Supervision. Dies wäre z. B. ein weiteres sinnvolles Angebot für CPDKurse.

\section{》) Wissen und praktische Kenntnisse sind nicht das Ende der professionellen Entwicklung}

Zwischen- und Abschlussprüfungen in der Weiterbildung müssen allerdings sanktionierenden Charakter haben. Aber sie müssen auch so aufgebaut werden, dass die Prüfer Wesentliches erfragen 
oder sich vorführen lassen und dass sie einheitlich, transparent, neutral und vergleichbar sind. Das notwendige Wissen kann durch Multiple-Choice-Fragen überprüft werden. Diese können, wie im Staatsexamen, Kenntnisse in funktioneller Anatomie und Pathophysiologie, Wissen über Indikationen und Kontraindikationen abfragen. Dazu bedarf es aber noch des konkreten, standardisierten Patienten, der vor den Augen von Prüfern befragt, untersucht und ggf. behandelt werden muss, damit das professionelle Verhalten erkennbar wird. Dieses Vorgehen ist bei den Ärztekammern in Deutschland nicht vorgesehen, auch wenn die Prüfer dort meist diejenigen sind, die auch in der Fort- und Weiterbildung aktiv sind.

Nach den Fortbildungskursen können Befragungen ohne sanktionierenden Charakter durchgeführt werden. Wichtiger ist hier die Erziehung zur Selbstreflexion: „Konnte ich mich in dieser Fortbildung in meinem Herangehen an den einzelnen Patienten verbessern?" Auch dafür haben wir noch keine Kultur entwickelt.

Dieses Heft soll einen Neuanfang darstellen, die Entwicklung eines kompetenten, professionellen Manualmediziners zu überdenken und unsere Strategien für die Fort- und Weiterbildung zu verbessern.

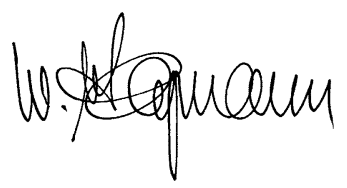

Dr. Wolfgang von Heymann

\section{Korrespondenzadresse}

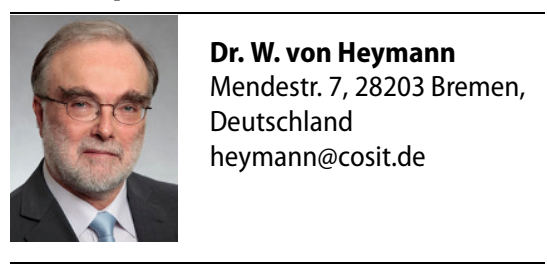

Interessenkonflikt. W. von Heymann gibt an, dass kein Interessenkonflikt besteht.

\section{Fragebogen für Skoliose-Patienten im deutschsprachigen Raum}

Wissenschaftler haben einen Fragebogen für den deutschsprachigen Raum verfügbar gemacht, mit dem die Lebensqualität von Patienten mit einer Skoliose erfasst werden kann. Damit wird die Therapie-Planung unterstützt.

Wie es Patienten mit Rückenleiden geht, ist nicht allein eine medizinische Frage. Ein wichtiger Faktor ist auch die von den Betroffenen empfundene Lebensqualität. Diesen Aspekt gilt es beispielsweise bei der Behandlung einer Skoliose zu berücksichtigen. Wissenschaftler um den Psychologen Dr. M. Thielsch von der Westfälischen Wilhelms-Universität Münster (WWU) und den Orthopäden Prof. Dr. T. Schulte von der Ruhr-Universität Bochum (St. Josef-Hospital) haben jetzt eine deutsche Übersetzung eines auf Spanisch, Englisch, Französisch und Griechisch etablierten Fragebogens zur Erfassung von Selbstwahrnehmung und Lebensqualität erstellt, namentlich "Quality of Life Profile for Spinal Disorders (QLPSD)“. Die deutsche Fassung des QLPSD haben die Forscher in einer Studie erprobt und für geeignet befunden. Damit steht Ärzten, Physiotherapeuten und anderen an der Behandlung beteiligten Experten nun ein neues deutschsprachiges Werkzeug zur Verfügung, mit dem sie sich ein genaues Bild vom Befinden von Patienten mit Skoliose machen können.

Skoliose betrifft rund drei bis fünf Prozent der Bevölkerung, wobei es viele Abstufungen des Schweregrades gibt. „Das Befinden der Patienten hängt einerseits vom Grad der Verkrümmung ab. Allerdings empfinden verschiedene Patienten selbst sehr ähnliche Deformitäten nicht unbedingt als gleichermaßen belastend - es hängt auch von der individuellen Verfassung und zahlreichen weiteren Faktoren ab. Im Röntgenbild ist also nicht zwingend zu erkennen, wie sich ein Patient fühlt", unterstreicht Schulte. „Der QLPSD füllt diese Lücke."

Die deutsche Version des QLPSD beruht auf dem bereits 1995 von spanischen Medizinern veröffentlichten Original. Mit ihr können die behandelnden Ärzte von ihren Patienten Informationen zu psychosozialen Problemen, Schlafqualität, Rückenschmerzen, Körperbild und Beweglichkeit innerhalb weniger Minuten standardisiert erfragen. Die deutsche Fassung zeichne sich durch eine hohe Messgenauigkeit und eine große Verlässlichkeit aus, so die Forscher.
Die Wissenschaftler befragten für ihre Studie 255 Patienten mit Skoliose sowie 189 gesunde Vergleichspersonen mit einem mittleren Alter von 30 Jahren. Sie verglichen die QLPSD-Ergebnisse mit den Resultaten von neun weiteren standardisierten Fragebögen zur Lebensqualität, zur Wahrnehmung des eigenen Körpers und zu psychischen Faktoren. Die Studie ist laut den Wissenschaftlern die weltweit größte wissenschaftliche Erprobung des QLPSD mit Skoliose-Patienten. Erstmals wurden nicht nur vorrangig Jugendliche, sondern auch Erwachsene bis ins hohe Alter systematisch untersucht.

"Ergibt die Auswertung des Fragebogens auffällig hohe Belastungswerte, sind Nachfragen angebracht. Gegebenenfalls sollte eine weitere Diagnostik und eine entsprechend angepasste Behandlung folgen, beispielsweise auch eine psychologische Unterstützung", sagt Thielsch. „Gerade ältere Patienten sowie Patienten mit schweren Skoliosen berichten über höhere Beeinträchtigungen der Lebensqualität aufgrund ihres Rückenleidens. Der QLPSD hilft, das Ausmaß abzuschätzen und die betroffenen Bereiche der Lebensqualität zu identifizieren."

Die Studienergebnisse wurden kürzlich in der Fachzeitschrift European Spine Journal online veröffentlicht. Die Forschungen zum QLPSD wurden bereits 2016 mit dem Nachwuchspreis der Deutschen Wirbelsäulengesellschaft e. V. geehrt.

Literatur:

Schulte T. L., Thielsch M. T. et al.: German validation of the quality of life profile for spinal disorders (QLPSD). European Spine Journal; First Online: 09 September 2017, DOI: 10.1007/s00586-017-5284-3

Quelle: Westfälische WilhelmsUniversität Münster, www.uni-muenster.de 